\title{
Applications of Polymeric Quantisation
}

\author{
Chen Jia Pern Neville ${ }^{1, *}$ and Ching Chee Leong ${ }^{1, * *}$ \\ ${ }^{1}$ National University of Singapore, Physics Department
}

\begin{abstract}
Polymer quantisation is a background independent quantisation scheme inspired by loop quantum gravity. Under this quantisation scheme, it predicts that space is discretised and changes in multiples of a fundamental length scale $\lambda$. As a result, the momentum operator is not well-defined. However, a new operator can be defined such that a Schrödinger-like equation can be retrieved. The solutions give rise to eigenspectra which are similar to the standard counterparts, with an additional correction term due to $\lambda$. We present the basic principles of the polymer representation and apply it to the harmonic oscillator to study the phenomenological implications of such solutions. In addition, we consider an ensemble of such oscillators and calculated the thermodynamical properties for systems that safisty the bosonic and fermionic statistics. The results presented may have physical significance at high energy scales or in exotic matter.
\end{abstract}

\section{Introduction}

Polymer quantisation is a quantisation scheme inspired by the methods of Loop Quantum Gravity (LQG) to quantise gravity in a background independent manner [1-3]. A crucial result produced by LQG is the introduction of a fundamental length scale $\lambda$ of which forms the 'quantum' of space. In other words, space is discretised into a structure similar to that of points on a lattice.Thus, the Hilbert space must be defined differently to accommodate new conditions. This results in an inequivalent representation - the so-called polymer representation. Naturally, the mathematical structure of the polymer representation is different, which may lead to novel physics.

We choose to explore the topic by looking at the statistical mechanics of polymer systems. One particular advantage of this method is that the systems that can be solved exactly under the polymer framework can be experimentally studied in laboratories, hence the bulk properties that arise due to a large collection of the polymer systems can be measured.

\section{Polymeric Quantisation}

We briefly set-up the formalism for polymeric quantisation in this section. It is a new representation of Quantum Mechanics, and is inequivalent to the Schrödinger representation familiar to physicists.

\footnotetext{
*e-mail: e0030993@u.nus.edu (corresponding author)

**e-mail: phyccl@nus.edu.sg
} 
Consider two generic translation operators $\hat{U}_{\mu}$ and $\hat{V}_{\lambda}$ with the following action on a wavefunction:

$$
\hat{U}_{\mu} \psi(p)=\psi(p-\mu) ; \quad \hat{V}_{\lambda} \psi(x)=\psi(x+\lambda) .
$$

If they fulfil the Heisenberg-Weyl algebra [4], defined by

$$
\begin{gathered}
\hat{U}_{\mu_{1}} \hat{U}_{\mu_{2}}=\hat{U}_{\mu_{1}+\mu_{2}} ; \quad \hat{V}_{\lambda_{1}} \hat{V}_{\lambda_{2}}=\hat{V}_{\lambda_{1}+\lambda_{2}} \\
\hat{U}_{\mu} \hat{V}_{\lambda}=e^{-i \mu \lambda / \hbar} \hat{V}_{\lambda} \hat{U}_{\mu},
\end{gathered}
$$

then the two operators form a pair of one-parameter unitary groups which are weakly continuous. By the Stone-von Neumann theorem [5], they are unitarily equivalent to the translation operators in the Schródinger representation:

$$
\hat{U}_{\mu}=e^{i \mu \hat{x} / \hbar} ; \quad \hat{V}_{\lambda}=e^{i \lambda \hat{p} / \hbar}
$$

In the polymer representation, the introduction of the discrete structure of space breaks the weak continuity of one of the operators. Thus, the violation of the Stone-von Neumann indicates that the polymer representation is indeed inequivalent to the Schrödinger representation.

The discrete structure of space can be visualised as a set of lattice points separated by a constant spacing $\lambda$. The basic kets in this Hilbert space are the position eigenkets $\left|x_{j}\right\rangle$. It can be shown that $\hat{V}_{\lambda}$ is no longer weakly continuous in $\lambda$, hence causing $\hat{p}$ to no longer be well-defined. This is a consequence of the fact that infinitesimal translations cannot occur. Thus, the basic operators become $\hat{x}$ and $\hat{V}_{\lambda}$, with the action

$$
\hat{x}\left|x_{j}\right\rangle=x_{j}\left|x_{j}\right\rangle ; \quad \hat{V}_{\lambda}\left|x_{j}\right\rangle=\left|x_{j}-\lambda\right\rangle .
$$

A key goal is to describe the time evolution of states, which is governed by the Schrödinger equation. However, the kinetic term requires $\hat{p}^{2}$, but $\hat{p}$ is not well-defined. A standard method in literature to introduce a momentum operator in the polymer representation $\hat{p}_{\lambda}$ is by approximating it through a trigonometric function:

$$
\hat{p}_{\lambda} \approx \frac{\hbar}{\lambda} \sin \left(\frac{\lambda \hat{p}}{\hbar}\right)=\frac{\hbar}{2 i \lambda}\left(\hat{V}_{\lambda}-\hat{V}_{\lambda}^{\dagger}\right) .
$$

This expression is motivated by the idea that $\hat{V}_{\lambda}$ is the only well-defined operator that is related to $\hat{p}$ and that $\hat{p}_{\lambda}$ should reduce to the canonical $\hat{p}$ as $\lambda \rightarrow 0$. Moreover, $\hat{p}$ is bounded between $-\frac{\hbar \pi}{\lambda}$ and $\frac{\hbar \pi}{\lambda}$ to give a unique value of the physical momentum $\hat{p}_{\lambda}$, which has a cut-off of $\frac{\hbar}{\lambda}$.

In an analogous fashion, we can define $\hat{p}_{\lambda}^{2}$ as

$$
\hat{p}_{\lambda}^{2} \approx \frac{\hbar^{2}}{\lambda^{2}}\left(2-2 \cos \left(\frac{\lambda \hat{p}}{\hbar}\right)\right)=\frac{\hbar^{2}}{\lambda^{2}}\left(2-\hat{V}_{\lambda}-\hat{V}_{\lambda}^{\dagger}\right) .
$$

Thus, the Hamiltonian operator is

$$
\hat{H}=\frac{\hbar^{2}}{2 m \lambda^{2}}\left(2-\hat{V}_{\lambda}-\hat{V}_{\lambda}^{\dagger}\right)+\hat{W}(x),
$$

where $\hat{W}(x)$ represents a generic potential term. 


\section{Applications of Polymeric Quantisation}

Solving the time-independent Schrödinger equation in (8) for various system will give the energy spectrum. We look at two important systems: the free particle and the harmonic oscillator [6].

The energy spectrum for a polymer free particle is

$$
E=\frac{\hbar^{2}}{2 m \lambda^{2}}\left(2-2 \cos \left(\frac{\lambda p}{\hbar}\right)\right),
$$

This spectrum is continuous but has a maximum value. In comparison with the standard dispersion relation, the polymer free particle has a negative first order correction that is proportional to $\lambda^{2}$.

For the harmonic oscillator, there is an exact solution to the energy spectrum but in the limit of interest $(\lambda \rightarrow 0)$, the energy spectrum reduces to

$$
E_{n}=\hbar \omega\left(\left(n+\frac{1}{2}\right)-\frac{\alpha^{2}}{32}\left(2 n^{2}+2 n+1\right)\right)
$$

which strongly resembles the energy spectrum of the harmonic oscillator, with a downwards correction proportional to $\alpha^{2} \equiv \frac{\lambda}{d}^{2}$ where $d$ is the characteristic length scale of the harmonic oscillator.

The two energy spectrum are sufficient to study different ensembles of particles, namely the free Bose and Fermi gases, and the blackbody radiation. In order to study the thermodynamics, we work with the grand canonical partition function $Z$ and compute it using the polymer modified density of states [7] given by

$$
\sum_{k} \rightarrow \frac{1}{h^{3}} \int \mathrm{d}^{3} \mathbf{r} \times \prod_{i=1}^{3} \int_{p_{i}=-\pi \hbar / \lambda}^{\pi \hbar / \lambda} \mathrm{d} p_{i} .
$$

Thus, the logarithm of the grand canonical partition functions are

$$
\ln Z=\left\{\begin{array}{l}
\frac{V}{\lambda_{\mathrm{th}}^{3}}\left(g_{\frac{5}{2}}(z)+\frac{3 m \lambda^{2} k_{B} T}{8 \hbar^{2}} g_{\frac{7}{2}}(z)\right)-\ln (1-z) \quad \text { (boson) } \\
\frac{V}{\lambda_{\mathrm{th}}^{3}}\left(f_{\frac{5}{2}}(z)+\frac{3 m \lambda^{2} k_{B} T}{8 \hbar^{2}} f_{\frac{7}{2}}(z)\right) \text { (fermion) }
\end{array}\right.
$$

where $\lambda_{\text {th }}$ is the thermal wavelength, and the $g_{\alpha}(z)$ and $f_{\alpha}(z)$ are known as the Bose-Einstein function and Fermi-Dirac function respectively [8]. Thus, we see that there are corrections to the thermodynamical variables which are proportional to $\lambda^{2}$, indicating that these corrections are typically minuscule.

For the Bose gas, the critical temperature at which Bose-Einstein condensation occurs undergoes a shift downwards. This shift is dependent on $\lambda$ :

$$
\lambda^{2}=\frac{\hbar^{2}}{k_{B} m} \frac{\zeta\left(\frac{3}{2}\right)}{\zeta\left(\frac{5}{2}\right)} \frac{\Delta}{T_{c}\left(T_{c}-\Delta\right)},
$$

where $\zeta(s)$ is the Riemann zeta function, and $\Delta$ is the shift in the critical temperature $T_{c}$. We can use this formula to estimate a value for $\lambda$ by treating $\Delta$ as the experimental uncertainty in our measurements of $T_{c}$. 
For the Fermi gas, the results of the thermodynamics can be used to study white dwarfs and neutron stars, which rely on the degeneracy pressure from the Pauli Exclusion Principle to support the immense inwards gravitational pressure. The pressure of a polymer electron gas is found to be smaller:

$$
P=\frac{1}{4} \rho E_{F}\left(1-\frac{m E_{F}}{16 \hbar^{2}} \lambda^{2}\right)
$$

where $E_{F}$ is the Fermi energy. Hence, it suggests that the Chandrasekhar limit $M_{C}$ is corrected downwards. It can be shown that the shift is proportional to $\lambda^{2}$ and takes the form

$$
\frac{M_{C}^{\lambda}}{M_{C}}=1-\frac{m E_{F}}{16 \hbar^{2}} \lambda^{2} .
$$

Measurements of the mass of white dwarfs can indeed help to estimate the bound on $\lambda$.

Finally, for the blackbody radiation, we used the modified harmonic oscillator energy spectrum in (10) to perturbatively express the density of states as

$$
g\left(\omega_{\lambda}\right)=\frac{V}{\pi^{2} c^{3}} \omega_{\lambda}^{3}\left(1+\frac{\alpha^{2} L}{8 \pi c} \omega_{\lambda}\right) .
$$

This allows us to find an expression for the Stefan-Boltzmann law in the polymer regime. It turns out that the Stefan-Boltzmann constant is dependent on the temperature and $\lambda$. The ratio

$$
\frac{\sigma_{\mathrm{SB}}^{\lambda}}{\sigma_{\mathrm{SB}}}=1+\frac{90 \zeta(5) \alpha^{2}}{\pi^{4}} \frac{L k_{B}}{h c} T
$$

allows for an estimation of $\lambda$ using measurements of the constant. Using the dimensions of the cavity in [9] and assuming that the harmonic oscillator length scale is on the order of the Bohr radius, the value for an upper bound for $\lambda$ is estimated to be $10^{-17} \mathrm{~m}$. This is a poor bound since it is only two orders of magnitude smaller than the radius of a nucleus, far higher than the proposed order of Planck's length.

\section{Conclusion}

We briefly reviewed the polymer framework and applied the results to the statistical thermodynamical properties of bosons, fermions, and the blackbody radiation. We found that the correction to the internal energy of the system due to the fundamental length scale is positive for all cases. The results presented offer a preliminary direction for experiments but at the current stage, it seems that even at the many-body level, the system is not sensitive to the effects of $\lambda$.

\section{References}

[1] A. Ashtekar, S. Fairhurst, J.L. Willis, Classical and Quantum Gravity 20, 1031 (2003)

[2] A. Ashtekar, J. Lewandowski, Classical and Quantum Gravity 21, R53 (2004)

[3] A. Corichi, T. Vukašinac, J.A. Zapata, Classical and Quantum Gravity 24, 1495 (2007)

[4] H. Weyl, Theory of Groups and Quantum Mechanics (Dover Publications Inc., 1950), ISBN 0486602699

[5] B. Hall, Quantum Theory for Mathematicians (Springer-Verlag GmbH, 2013), ISBN 146147115X 
[6] A. Corichi, T. Vukašinac, J.A. Zapata, Phys. Rev. D 76, 044016 (2007)

[7] M.A. Gorji, K. Nozari, B. Vakili, Phys. Rev. D 90, 044051 (2014)

[8] R.K. Pathria, P.D. Beale, Statistical Mechanics (Elsevier LTD, Oxford, 2011), ISBN 0123821886

[9] W.R. Blevin, W.J. Brown, Metrologia 7, 15 (1971) 